\title{
COMORBIDADES DO TRANSTORNO DE DÉFICIT DE ATENÇÃO E HIPERATIVIDADE EM CRIANÇAS ESCOLARES
}

\author{
Marianne de Aguiar Possa', Lucas Spanemberg ${ }^{2}$, Ana Guardiola ${ }^{3}$
}

\begin{abstract}
RESUMO - Objetivo: Avaliar a freqüência de transtorno de conduta (TC), transtorno desafiador opositivo (TDO) e transtorno obsessivo-compulsivo (TOC) em crianças com transtorno de déficit de atenção e hiperatividade (TDAH). Método: Estudo realizado em crianças de 7 a 11 anos com TDAH conforme critérios do DSM-IV ( $n=35)$, exame neurológico normal e exame neurológico evolutivo (ENE) segundo Lefèvre alterado. Os responsáveis responderam a questionários contendo os critérios do DSM-IV para TDAH e para comorbidades. Resultados. TDAH do tipo combinado foi o mais prevalente $(51,4 \%)$. Quatorze crianças $(40,0 \%)$ apresentaram TC, incluindo duas que apresentaram TC e TOC. Cinco (14,2\%) apresentaram apenas TDO e uma (2,8\%) apresentou apenas TOC. Onze das quatorze crianças $(78,5 \%)$ com TC tinham TDAH do tipo combinado $(p<0,05)$. Onze das quinze crianças $(73,3 \%)$ sem comorbidades eram do tipo desatentas ou hiperativas $(p<0,05)$. Conclusão:O TDAH a presenta alta freqüência de comorbidade com TC, que parece estar preferencialmente associado ao TDAH combinado.
\end{abstract}

PALAVRAS-CHAVE: transtorno de déficit de atenção e hiperatividade, comorbidades, crianças.

\begin{abstract}
Attention-deficit hyperactivity disorder comorbidity in a school sample of children
ABSTRACT - Objective: To evaluate the frequency of conduct disorder (CD), oppositional defiant disord er (ODD) and obsessive-compulsive disorder (OCD) in children with attention-deficit hyperactivity disorder (ADHD). Method: This study was performed with children between 7 and 11 years old who fit the DSMIV diagnostic criteria $(n=35)$ to ADHD, normal neurologic examination and abnormal evolutionary neuro logical examination (ENE) by Lefèvre. Parents answered a questionnaire with DSM-IV criteria to ADHD and comorbidities. Result.ADHD combined subtype was the most prevalent (51.4\%). Fourteen (40\%) had CD, including two with both CD and OCD. Five (14.2\%) had only ODD and one (2.8\%) only OCD. Eleven of fourteen children (78.5\%) with CD had also ADHD combined subtype, with significant statistical difference $(p<0.05)$. Eleven of fifteen children $(73.3 \%)$ without comorbidity had inattentive or hyperactive subtype $(p<0.05)$. Conclusion: The frequency of association between $C D$ and ADHD was high, much more in combined subtype.
\end{abstract}

KEY WORDS: attention-deficit hyperactivity disorder, comorbidity, children.

O transtorno de déficit de atenção e hiperatividade (TDAH) é condição comum em crianças e re sponsável por uma série de prejuízos médico-sociais, especialmente dificuldades escolares ${ }^{1-3}$. É classicamente caracterizado por alterações dos sistemas motores, perceptivos, cognitivos, de comportamento, comprometendo o aprendizado de crianças com potencial intelectual adequado ${ }^{1,4-6}$. A Associação Americana de Psiquiatria, através do DSM-IV o define como um padrão persistente de desatenção e/ou hiperatividade, mais freqüente e severo do que aquele tipicamente observado em indivíduos em nível equivalente de desenvolvimento e associado a algum prejuízo devido aos sintomas em pelo menos dois contextos, como casa, escola ou trabalho ${ }^{7}$. Este transto mo tem recebido designações diferentes ao longo do tempo. Strauss e Lehtinen em 1947 o denominaram "lesão cerebral mínima". Em um simpósio o c o rrido em Oxford em 1962, a expressão "disfunção c e rebral mínima" foi oficializada8. Em 1968, o DSM-II descreveu o transtorno como caracterizado por "níveis excessivos de atividade". O DSM-III de 1980 ampliou o foco do transtorno para incluir desatenção e impulsividade em adição à hiperativi-

Departamento de Neurologia e Neuro ci ru rgia da Faculdade de Ciências Médicas de Porto Alegre (FFFCMPA) e Serviço de Neurologia da Irmandade Santa Casa de Misericórdia, Porto Alegre - RS, Brasil: ${ }^{1}$ Médica residente do Serviço de Psiquiatria do Hospital das Clínicas de Porto Alegre; ${ }^{2}$ Médico residente do Serviço de Psiquiatria do Hospital São Lucas da Pontifícia Universidade Católica do Rio Grande do Sul; ${ }^{3}$ Professora Adjunta de Neurologia da FFFCMPA, Doutora Livre-Docente em Neurologia Infantil pela FFFCMPA.

Recebido 15 Setembro 2004, recebido na forma final 26 Novembro 2004. Aceito 26 Janeiro 2005.

Dra. Marianne de Aguiar Possa - Rua Carlos Von Koseritz 756/305 - 90540-030 Porto Alegre RS - Brasil.

E-mail: mariannepossa02@yahoo.com.br 
dade 9 . Assim, foi criada a expressão "transtorno de déficit de atenção com ou sem hiperatividade" pela Associação Psiquiátrica Americana. Na revisão deste manual em 1987, a designação foi alterada para "transtorno de déficit de atenção/hiperatividade" e mantida na edição atual, o DSM-IV8.

O TDAH tem prevalência estimada em torno de 3,5\% a $8 \%$ em diferentes áreas geográficas incluindo o Brasil ${ }^{2,8-10}$. A prevalência é mais alta no meio urbano em relação ao meio rural e aumenta em função de condições sócio-econômicas mais precárias. Muitos estudos apontam para maior prevalência no sexo masculino ${ }^{11}$. Tem sido associado com fatores genéticos e ambientais. A exposição materna ao álcool e/ou tabagismo durante a gestação e depressão materna podem ser fatores predisponentes ao transtorno ${ }^{12}$. Os valores de prevalência podem variar conforme a faixa etária pesquisada (pré-escolares, escolares $)^{13}$ ou os critérios diagnósticos utilizados (critérios do DSM-IV, entrevista clínica ou estruturada, critérios neuropsicológicos, ou outros). Foi demonstrado que os critérios do DSM-IV superestimam a prevalência do transtorno, provavelmente por uma baixa especificidade do método e, portanto, tem melhor aplicabilidade como exame de rastreamento pela praticidade e alta sensibilidade ${ }^{8}$.

Estudos de comorbidade revelaram taxas elevadas de ocorrência com outros transtornos psiquiátricos, em especial os transtornos do humor, transtornos ansiosos e os transtornos disruptivos do comportamento ${ }^{13,14}$. Na associação do TDAH com o u trostranstornos, além das dificuldades causadas pela soma de sintomas de mais um transtorno, há também um aumento na prevalência de complicações em relação ao TDAH isolado. Por exemplo, o TDAH associado a transtorno afetivo bipolar e transtorno de conduta, predispõe ao abuso de substâncias na adolescência, enquanto o TDAH isolado é um fraco fator de risco para abuso de substâncias². O diagnóstico pelo DSM-IV, por ser pouco específico, carrega alta taxa de comorbidade, que ocorrem principalmente com transtorno de conduta e transtorno desafiador opositivo ${ }^{15-16}$. Entretanto, o estudo de genética do National Institute of Mental Health demonstrou de forma clara que os sintomas de TDAH não são meramente expressão do quadro clínico de outros transtornos e representam uma comorbidade real ${ }^{16}$. A apresentação clínica do TDAH pode ser predominantemente desatento, $p$ redominantemente hiperativo, ou combinado. A maioria das crianças e adolescentes apresentam o tipo combinado. Os pacientes do tipo desatento são geralmente do sexo feminino e de maior faixa etária.
O tipo de menor faixa etária é o hiperativo. Os pacientes do tipo combinado têm maior prejuízo global do que os outros dois tipos. Os tipos combinado e desatento têm maiores índices de fracasso escolar do que o tipo hiperativo ${ }^{17,18}$. O objetivo deste estudo é avaliar a comorbidade dos transtornos de conduta (TC), obsessivo-compulsivo (TOC) e desafiante opositivo (TDO) em amostra escolar com diagnóstico de TDAH segundo os critérios do DSM-IV. Serão analisadas ainda as comorbidades conforme o gênero.

\section{MÉTODO}

Este estudo consiste em uma análise observacional, de delineamento transversal realizada em 3 escolas estaduais e 1 municipal de Porto Alegre com alunos do ensino fundamental entre outubro de 2002 e maio de 2003. Foram incluídas no estudo 35 crianças de ambos os sexos e idades entre 7 e 11 anos que apresentaram TDAH conforme critérios do DSM-IV.

Para a seleção da amostra, os professores das escolas receberam um protocolo contendo características de comportamento compatíveis com TDAH de acordo com o DSM-IV e selecionaram seus alunos que poderiam apresentar o distúrbio, para fins de rastreamento. Este questionário continha 15 das 18 questões do critério A do DSMIV. Aqueles que apresentassem 8 questões positivas eram encaminhados para a próxima etapa. Em seguida, as crianças selecionadas, receberam visita em suas casas pelos autores para a aplicação de questionários mais detalhados contendo critérios $A, B, C, D$ e E do DSM-IV para TDAH A, B e C para TC; A, B, C e D para TDO, e A, B, C e D para TOC. Esses questionários foram aplicados aos pais ou responsáveis maior de 18 anos, após este estar de acordo e assinar o termo de consentimento informado. Por último, as crianças foram examinadas nas escolas, individualmente pelos autores, através de exame neurológico tradicional e exame neurológico evolutivo (ENE) de Lefèvre ${ }^{5}$. Foram incluídas na amostra as crianças que apresentaram exame neurológico tradicional normal e exame neurológico evolutivo alterado.

O projeto de pesquisa foi aprovado pelo Comitê de Ética em Pesquisa da ISCMPA.

O teste exato de Fisher foi utilizado para determinar dife rença significativa entre as variáveis categóricas e foi considerada diferença estatística se $p<0,05$.

\section{RESULTADOS}

Foi diagnosticado TDAH segundo critérios do DSM-IV em 35 crianças escolares da rede pública da cidade de Porto Alegre. Houve predomínio do sexo masculino na amostra embora a associação de TDAH com o sexo masculino não tenha sido comp rovada estatisticamente (Tabela1). O tipo combinado de TDAH foi predominante na amostra (Tabela 2 ), seguido dos tipos desatento e hiperativo em 
Tabela 1. Dados demográficos da amostra $(n=35)$.

\begin{tabular}{lc}
\hline $\begin{array}{l}\text { Média de idade (anos) } \pm \text { DP } \\
\text { Sexo }\end{array}$ & $8,77 \pm 1,45$ \\
Masculinos & $20(57,1 \%)$ \\
Femininos & $15(42,9 \%)$ \\
Cor & \\
Brancos & $17(48,6 \%)$ \\
Negros & $10(28,6)$ \\
Outros/ mestiços & $8(22,9 \%)$ \\
Tipo de escola & \\
Estadual & $27(77,1 \%)$ \\
Municipal & $8(22,9 \%)$ \\
Série (ensino fundamental) & \\
$1^{\text {a }}$ & $13(37 \%)$ \\
$2^{\text {a }}$ & $7(20 \%)$ \\
$3^{\text {a }}$ & $11(31,4 \%)$ \\
$4^{\text {a }}$ & $4(11,4 \%)$ \\
\hline DP, Desvio-padrão (anos)
\end{tabular}

DP, Desvio-padrão (anos)

Tabela 2. Distribuição entre os sexos e tipos de TDAH.

\begin{tabular}{lccc}
\hline & $\begin{array}{c}\text { Masculino } \\
\mathrm{N}(\%)\end{array}$ & $\begin{array}{c}\text { Feminino } \\
\mathrm{N}(\%)\end{array}$ & $\begin{array}{c}\text { Total } \\
\mathrm{N}(\%)\end{array}$ \\
\hline TDAH hiperativo & $1(2,8)$ & $3(8,5)$ & $4(11,4)$ \\
TDAH desatento & $8(22,8)$ & $5(14,2)$ & $13(37,1)$ \\
TDAH combinado & $11(31,4)$ & $7(20,0)$ & $18(51,4)$ \\
Total & $20(57,1)$ & $15(42,8)$ & $35(100,0)$ \\
\hline Não houve diferença significativa $(\mathrm{p}>0,05)$ pelo teste exato de Fischer
\end{tabular}

Tabela 3. Distribuição das comorbidades conforme tipo de TDAH.

\begin{tabular}{lccc}
\hline Diagnóstico & $\begin{array}{c}\text { Hiperativo }(\mathrm{H}) \text { ou } \\
\text { Desatento }(\mathrm{D})\end{array}$ & Combinado & Total \\
\hline Apenas TDAH & $11 *(8 \mathrm{D}+3 \mathrm{H})$ & 4 & 15 \\
TDAH+TC & $3(3 \mathrm{D})$ & $11 *$ & 14 \\
TDAH+TDO & $2(2 \mathrm{D})$ & 3 & 5 \\
TDAH+TOC & $1(1 \mathrm{H})$ & $2 \mathrm{a}$ & 3 \\
\hline
\end{tabular}

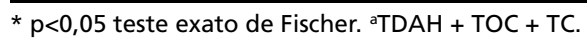

o rdem decrescente de freqüências. Embora o sexo masculino tenha sido predominante entre as crianças com TDAH do tipo combinado e desatento, essa associação não foi estatisticamente significativa

A maioria das crianças apresentou alguma das comorbidades estudadas (Tabela 3). O TC, seguido do TDO, foi a comorbidade mais prevalente, englobando $40 \%$ da amostra (14 casos), que inclui também 2 casos de TC e TOC. O tipo combinado foi o mais freqüente na amostra com TC, com 11 casos com TDAH combinado e 3 com TDAH desatento. A associação de TC com TDAH do tipo combinado foi estatisticamente significativa. As crianças desatentas

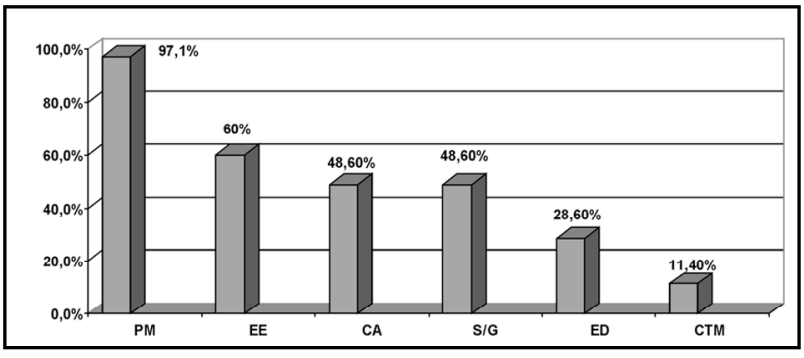

Gráfico. Freqüência de alterações no exame neurológico evolu tivo de Lefévre. $P M$, persistência motora; $E E$, equilíbrio estáti Co; CA, coordenação apendicular; S/G, sensibilidade e gnosias; $E D$, equilíbrio dinâmico; CTM, coordenação tronco e membros.

ou hiperativas mais freqüentemente apresentaram TDAH isolado, sem comorbidades. Apenas 4 casos de TDAH sem outros diagnósticos compreendiam crianças com TDAH combinado, as 11 restantes eram do tipo desatento ou hiperativo. Essa associação foi comprovada estatisticamente.

Foram considerados casos de TDO somente aqueles que não preenchiam os critérios diagnósticos para TC. A presença de TC exclui o diagnóstico de TDO, segundo as diretrizes diagnosticas do DSMIV, são portanto diagnósticos que jamais aparecem juntos. O TOC foi a comorbidade menos prevalente com apenas 3 casos $(8,5 \%)$. Duas crianças com TDAH a p resentaram TOC e TC e 1 apenas com TOC como comorbidade.

Nenhuma criança apresentou alterações no exame neurológico tradicional e todas apresentaram alguma alteração no ENE. As alterações mais f reqüentes foram principalmente nas provas de persistência motora com 34 casos $(97,1 \%)$ e do equilíbrio estático, seguidas das alterações na coordenação apendicular, sensibilidade e gnosias, equilíbrio dinâmico e coordenação tronco-membros (Grá fico). Além disso, 27 (77,1\%) tinham dificuldades de alfabetização, $6(17,1 \%)$, lateralidade mal estabelecida e $2(5,7 \%)$, dislalias.

\section{DISCUSSÃO}

Nossos resultados demonstram predomínio do TDAH no sexo masculino ( $57,1 \%$ vs. $42,9 \%)$, embora essa diferença não seja estatisticamente significativa. Esses resultados são compatíveis com alguns estudos, que mostram maior prevalência no sexo masculino ${ }^{8,13,19}$ embora outros autores não confirmem esse predomínio ${ }^{2}$.A maior freqüência de TDAH em meninos pode ser explicada por diferenças na a p resentação do transtorno em relação ao gênero. Newcorn et al. ${ }^{20}$, em um estudo sobre perfil dos sintomas e comorbidades em função do gênero, en- 
controutaxas menores de impulsividade em meninas comparadas a meninos, resultado também encontrado por outros autore $s^{9,21,22}$. Além disso, tanto pais quanto professores relataram que meninas com TDAH são menos sintomáticas que meninos ${ }^{20}$. Dalsgaard et al. ${ }^{19}$, em estudo de coorte, encontraram maior associação de admissões psiquiátricas e sexo feminino ao longo de 20 anos. Portanto, apesar de menos sintomáticas, meninas com TDAH podem ter pior prognóstico.

Neste estudo, o tipo combinado de TDAH se mostrou predominante na amostra com 18 casos $(51,4 \%)$, seguido pelos tipos desatento e hiperativo, resultados semelhantes aos de outros estudos ${ }^{17,18,23}$. Ao contrário de alguns autores, não houve pre dominância de sexo masculino no tipo hiperativo ${ }^{9}$ ou feminino no tipo desatento ${ }^{17,18}$. A amostra reduzida deste estudo pode ter limitado estas conclusões já que apenas 4 casos de crianças com o tipo hiperativo foram encontradas.

Transtomos psiquiátricos comórbidos são comuns no $\mathrm{TDAH}^{19}$, part i c u l a rmentetranstornos disruptivos, de humor e de ansiedade ${ }^{9,13,21-23}$. Nesta amostra, a maioria das crianças com TDAH $(57,14 \%)$ manifestava alguma das comorbidades estudadas, em concordância com outros estudos ${ }^{13,16,23}$. Os transtornos disruptivos, TC e TDO, foram amplamente $p$ revalentes em nosso estudo. Mais da metade das crianças com TDAH $(54,2 \%)$ apresentou TC ou TDO, resultado semelhante ao encontrado na revisão de Plizka ${ }^{24}$ e no estudo de Souza et al. ${ }^{16}$. Rohde et al. ${ }^{2}$, em estudo com amostra de perfil semelhante (comunitária), encontrou também taxas equivalentes, com $47,8 \%$ dos escolares reunindo critérios para transtornos disruptivos. Pacientes com TDAH associado a TC têm maior risco de apresentar abuso ou dependência de substâncias psicoativas de uso ilícito, maior dificuldade de adesão ao tratamento, e também apresentam mais fatores confundidores para uma avaliação da adequação ao tratamento, apresentando, portanto, pior prognóstico e mais difícil manejo ${ }^{25}$.

Nesta pesquisa, crianças com TDAH tipo combinado foram significativamente $(p<0,05)$ mais $p$ re dispostas a apresentar TC, com 11 (78,57\%) dos 14 casos de TC pertencentes a este grupo. Das 15 crianças que não apresentaram as comorbidades estudadas, apenas 4 eram do tipo combinado; as 11 (73,33\%) restantes eram do tipo desatentas ou hiperativas $(p<0,05)$. Esses dados são importantes clinicamente pois a correta caracterização em subtipos do TDAH poderia conduzir a investigações diagnósticas mais cuidadosas nas crianças com maior risco para determinada comorbidade. Eiraldi et al. ${ }^{9}$, ao estudarem padrões de comorbidade em relação a subtipos de TDAH (TDAH combinado e TDAH desatento), encontraram maiores taxas de TC e TDO em crianças com TDAH do tipo combinado do que nas do tipo desatento. Ambos os grupos apresentavam maiores taxas de TC, TDO, transtornos de ansiedade (TA) e de humor do que o grupo controle. O tipo combinado parece ser uma forma mais severa de TDAH comparado aos outros subtipos, em relação às comorbidades $^{17}$.

Além do efeito aditivo na sintomatologia das crianças com TDAH, a comorbidade também poderia ter um impacto sobre o núcleo de sintomas de TDAH $^{20}$. Por exemplo, crianças com TDAH e TC ou TDO seriam mais impulsivas que crianças com TDAH sem comorbidades ou com TDAH e TA. Isto é consistente com a hipótese de Plizka, segundo a qual a comorbidade com TA mitiga a impulsividade em crianças com TDAH. Por outro lado, crianças com TDAH, TA e TC ou TDO são similares quanto à imputsividade àquelas com TDAH e TC ou TDO sem TA. Nesses casos o TA não mitiga os sintomas de hiperatividade e impulsividade em crianças com TDAH. Isso mostra que o núcleo psicopatológico em indivíduos com transtornos comórbidos pode mudar quando uma condição adicional é acrescentada ou subtraída da combinação ${ }^{20}$. Outro exemplo é a inte ração da comorbidade em relação a resposta ao tratamento. Enquanto os transtornos disruptivos têm poderosos efeitos nas características de base do TDAH, isso raramente interage com a resposta ao tratamento. Em contraste, o TA exerce efeitos mais discretos nas características de base, mas freqüentemente interage com a resposta a tratamentos específicos (crianças com TDAH e TA respondem melhor ao tratamento). Portanto, os perfis clínicos totais dos diferentes tipos de comorbidades seriam suficientemente distintos para dar suporte à necessidade de discriminação entre os grupos ${ }^{22}$. Propostas de inclusão de novos subtipos de TDAH em relação a suas comorbidades (TDAH subtipo agressivo vs. TDAH subtipo ansioso) aguardam uma oficialização ${ }^{21}$. Modelos integrativos serão necessários em estudos futuros. Somente 3 crianças $(8,57 \%)$ desta amostra tiveram comorbidade com TOC, sendo que em somente um caso, o TOC era a única comorbidade (nos outros dois, TDAH e TOC eram acompanhados por TC). Já foi demonstrado que quando TDAH é visto em jovens com TOC, estas duas entidades refletem comorbidades verdadeiras, e não apenas 
artefatos de sintomatologia secundária ${ }^{26}$. Identificar a comorbidade nestas crianças pode ser fundamental para o sucesso do tratamento.

Em conclusão, nesta pesquisa, o tipo combinado de TDAH se mostrou predominante, seguido pelos subtipos desatento e hiperativo. A maioria das crianças com TDAH apresentou alguma comorbidade psiquiátrica, sendo TC e TDO as mais prevalentes. Portanto, salienta-se a necessidade de, diante de um caso de TDAH, a pesquisa de morbidades associadas.

Agradecimentos - Ao Sr. Alan Birck pela extrema dedicação e paciência no auxílio à análise estatística dos dados deste trabalho.

\section{REFERÊNCIAS}

1. Guardiola A. Uso da imipramina no estudo de hiperatividade com déficit de atenção. Tese de Mestrado Porto Alegre. Fundação Faculdade Federal de Ciências Médicas de Porto Alegre, 1990.

2. Rohde LA, Biederman J, Busnello EA, et al. ADHD in a school sample of Brazilian adolescents: a study of prevalence, comorbid conditions, and impairments. J Am Acad Child Adolesc Psychiatry 1999;38:716-722.

3. Doyle AE, Faraone SV, DuPre EPBA, Biederman J. Separating attention deficit hyperactivity disorder and learning disabilities in girls: a familial risk analysis. Am J Psychiatry 2001;158:1666-1672.

4. Rebollo MA. Etiologia de la disfunción cerebral mínima. Montevidéo: Neuropediatria Latinoamericana, 1972.

5. Lefèvre AFB. Exame neurológico evolutivo,. 2a ed. São Paulo: Sarvier, 1977.

6. Fischer W, Burd L, Kuna D, Berg D. Attention deficit disorders and hyperactivity in multiple disable children. Rehabilitation Literature 1985; 46:250-254.

7. Associação Psiquiátrica Americana. Manual de diagnóstico e estatística de distúrbios psiquiátricos,. 4 ed. (DSM-IV). Porto Alegre: Artes Médicas, 1994.

8. Guardiola A, Fuchs FD, Rotta NT. Prevalence of attention-deficit hyperactivity disorder in students: comparison between DSM-IV and neuropsychological criteria. Arq Neuropsiquiatr 2000;58:401-407.

9. Eiraldi RB, Power TJ, Nezu CM. Patterns of comorbidity associated with subtypes of attention-deficit/ hyperactivity disorder among 6- to 12-yearold children. J Am Acad Child Adolesc Psychiatry 1997;36:503-514.

10. Guardiola A. Distúrbio de hiperatividade com déficit de atenção: um estudo de prevalência e fatores associados em escolares Tese de Douto- rado. Porto Alegre. Faculdade de Medicina da Universidade Federal do Rio Grande do Sul, 1994.

11. Wender EH. Attention-deficit/hyperactivity disorder: is it common? Is it overtreated?. Arch Pediatr Adolesc Med 2002;156:209-210.

12. MickE, Biederman J, Faraone SV, Sayer J, Kleinman MSW. Case-control study of attention-deficit hyperactivity disorder and maternal smoking, alcohol use, and drug use during pregnancy. J Am Acad Child Adolesc Psychiatry 2002;41:378-385.

13. Wilens TE, Biederman J, Brown S, et al. Psychiatry comorbidity and functioning in clinically referred preschool children and school-age youths with ADHD. J Am Acad Child Adolesc Psychiatry 2002;41:262-268.

14. Biederman J, Newcorn J, Sprich S. Comorbidity of attention deficit hyperactivity disorder with conduct, depressive, anxiety and other disorders. Am J Psychiatry 1991;148:564-577.

15. Arnold LE, Jensen PS. Transtorno do déficit de atenção. In: Kaplan HI, Sadock BJ (eds). Tratado de Psiquiatria, 6 ed. Porto Alegre: Artes Médicas; 1999;2495-2511.

16. Souza I, Serra MA, Mattos P, Franco VA. Comorbidade em crianças e adolescentes com transtorno do déficit de atenção: resultados preliminares. Arq Neuropsiquiatr 2001;59:401-406.

17. Faraone SV, Biederman J, Weber W, Russel RL. Psychiatric, neuropsychological, and psychosocial features of DSM-IV subtypes of attention-deficit/ hyperactivity disorder: results from a clinically referred sample. J Am Acad Child Adolesc Psychiatry 1998;37:185-193.

18. Faraone SV , Biederman J, Mick E, et al. A family study of psychiatry comorbidity in girls and boys with attention-deficit/hyperactivity disorder. Biol Psychiatry 2001;50:586-592.

19. Dalsgaard S, Mortensen PB, Frydenberg M, Thomsen PH. Conduct problems, gender and adult psychiatry outcome with attention-deficit hyperactivity disorder. Br J Psychiatry 2002;181:416-421.

20. Newcorn JH, Halperin JM, Jensen PS, et al. Symptom profiles in children with ADHD: effects of comorbidity and gender. J Am Acad Child Adolesc Psychiatry 2001;40:137-146.

21. Jensen PS, Martin D, Cantwell DP. Comorbidity in ADHD: implications for research, practice, and DSM-V. J Am Acad Child Adolesc Psychiatry 1997;38:1065-1079.

22. Jensen PS, Hinshaw SP, Kraemer HC et al. ADHD comorbidity findings from the MTA study: comparing comorbid subgroups. J Am Acad Child Adolesc Psychiatry 2001;40:147-158.

23. Busch B, Biederman J, Cohen LG, et al. Correlates of ADHD among children in pediatric and psychiatric clinic. Psychiatry Services 2002;3:1103-1111.

24. Plizka SR. Comorbidity of attention- deficit/hyperactivity disorder with psychiatric disorder: an overview. J Clin Psychiatry 1998;59:(Suppl 7):S50-S58.

25. Rohde LA, Mattos P. Princípios e práticas em transtorno de déficit de atenção/hiperatividade. Porto Alegre (RS): Artmed; 2003.

26. Geller DA, Biederman J, Faraone SV et al. Attention-deficit/ hyperactivity disorder in children and adolescents with obsessive-compulsive disorder: Fact or artifact? J Am Acad Child Adolesc Psychiatry 2002;41:52-58. 